\title{
Non-physics peer demonstrators in undergraduate laboratories: a study of students' perceptions
}

\author{
Michael Braun ${ }^{1} \ddagger$ and Les Kirkup ${ }^{1}$ \\ ${ }^{1}$ School of Mathematical and Physical Sciences, University of Technology Sydney
}

\begin{abstract}
Laboratory demonstrators play a crucial role in facilitating students' learning in physics subjects. Inspired by the success of peer-led activities, we introduced peer demonstrators to support student learning in first-year physics subjects enrolling students not intending to major in physics. Surveys were administered to 1700 students over 4 years in 4 subjects to examine, for example, student perceptions of how well demonstrators were prepared to assist them in the laboratory. Scores awarded to peer demonstrators by students were no lower than those awarded to demonstrators traditionally employed in the first year physics laboratory. These latter demonstrators were drawn mainly from the ranks of physics research students. The findings validate the recruitment of peer demonstrators and will be used to inform the recruitment and support programs for laboratory demonstrators.
\end{abstract}

PACS numbers: 01.50.Qb, 01.40.gb, 01.40.Fk

Keywords: demonstrator, teaching assistant, laboratory class, peer learning

$\ddagger$ Present address: School of Mathematical and Physical Sciences, Faculty of Science, University of Technology Sydney, Broadway, NSW 2007, Australia 
Non-physics peer demonstrators in undergraduate laboratories: a study of students' perceptions2 Submitted to: Eur. J. Phys. 
Non-physics peer demonstrators in undergraduate laboratories: a study of students' perceptions3

\section{Introduction}

The practical component is a core element of science courses often intended to develop experimental skills (Psillos and Niedderer 2002); in some cases serving to reinforce theoretical principles and concepts (Von Aufschnaiter and Von Aufschnaiter 2007), in

others employed to develop investigative and communication skills (Seifert et al. 2009). Laboratory-based activities feature prominently in science courses. For example, in first year physics subjects at University of Technology Sydney, laboratory-based classes account for about a third of the student's total contact time. This is true of physics subjects forming part of physical science majors as well as other science and engineering majors. In these subjects, students may see a succession of lecturers and tutors through the semester but they will generally stay with the same demonstrators. As a result, the contact time with a demonstrator may be 2 to 3 times greater than the time spent with any other individual academic staff. Finally, the laboratory class size is typically smaller than the lecture class. These factors point to the important role the demonstrators play in the learning of science. The importance of demonstrators in further underlined by a national report (Rice et al. 2009), which noted that demonstrators had the power to make a lab a great or a miserable experience.

In the conventional approach, first year physics practical classes are staffed with demonstrators (sometimes referred to as teaching assistants) largely drawn from the ranks of research students, postdoctoral fellows, and non-tenured part-time academics (Jaeger 2008). For the research student/postdoctoral fellow, demonstrating serves provide valuable experience especially for those contemplating an academic career, and to supplement income. For the faculty, on-site availability and flexibility offered by such demonstrators assists in staffing the laboratory classes and facilitates closer interactions between the subject coordinator and the demonstrators.

The majority of students are enrolled in non-physics majors and degrees, have different career goals, different epistemological stances (Roth and Roychoudhury 1994), and are generally younger than their demonstrators. Kirkup et al. (2010) postulated that the academic, generational and social separation between the teacher and the student affects the learning experience and efficacy of the class.

The well-known peer instruction model (Crouch and Mazur 2001), originally devised for introductory physics courses, attempts to bridge the gap especially in large-size lectures, where nearby students act as interlocutors in short debates over interpretations of concepts. Motivated also to enhance cognitive engagement, similar interactions with fellow students have been explored in a physics laboratory setting (Cox and Junkin III 2002, Jarrett et al. 2010). Outside formal classes, advantages of peer learning in study group activities, as exemplified by Supplemental Instruction (USA), Peer-Assisted Learning (UK) and Peer-Assisted Study Sessions (Australia), are well established (Dawson et al. 2014). Although the use of peer leaders in these programs was initially motivated by the desire to distinguish the programs from formal instruction and to distance them from remedial classes (Arendale 2002), the greater proximity of 
Non-physics peer demonstrators in undergraduate laboratories: a study of students' perceptions4

the peer leader to the learner in terms of age and career aspirations contributed to the success of the programs.

At UTS, encouraged by the reported success of peer learning activities (Hensen and Shelley 2003), we gradually introduced peer demonstrators to physics practical classes in first-year subjects supporting engineering and life science courses. The peer demonstrators are drawn from those who recently completed the subject with outstanding results. The exposure of peer demonstrators to formal post-secondary physics coursework is limited to that subject. The selection follows an interview where the candidate's suitability for the position is evaluated, based on criteria that include included evidence of high-order communications skills, relevant experience, response to hypothetical scenarios, and appreciation of the inquiry-based approach. Kirkup et al. (2010) found differences between physics-based, experienced, demonstrators and "senior students" (from whose ranks peer demonstrators are drawn) in the emphasis on professional relevance and the outcomes of inquiry-oriented practicals. Kendall and Schussler (2012) documented differences in the student perception of the tenured staff (knowledgeable but distant) and graduate teaching assistants (lacking confidence but relatable). Students' and teachers' differing perceptions of the relative importance of certain teaching practices (Zepke et al. 2014) may be better aligned in the case of peer demonstrators as their background, experience and career trajectory is likely to be closer to the students'. Peer demonstrators may help bridge barriers to student engagement although the tendency to perpetuate teaching practices experienced during the teachers' undergraduate studies may need to be overcome through a suitable professional development program (Roehrig et al. 2003).

In this paper, we evaluate the effect of the introduction of non-physics major peer demonstrators on the student perception of the demonstrator-student interactions in the laboratory. Towards this end, we propose a separation measure to categorize the levels of

background dissimilarity between the students and the demonstrators, and analyze the differences in survey responses of students to the different categories of demonstrators.

\section{Method}

We focused on laboratory classes in first-year physics subjects, each enrolling in the range of 200 to 600 students a year: "Physical Modelling", offered to engineering students, "Physical Aspects of Nature", offered to students in medical science, biomedical science, environmental science, and allied courses (to be referred to jointly as the life science cohort), and "Foundations of Physics" (in the first semester) and "Physics in Action" (in the second semester), offered to physics, nanotechnology, chemistry, and related courses (to be referred to as the physical science cohort). In these subjects, the laboratory classes numbered no more than 40 students each and were staffed by two demonstrators, a principal and an assistant demonstrator.

For most of the time, particularly in terms of interactions with individual students or small student teams, the roles of the principal demonstrator and the assistant 
Non-physics peer demonstrators in undergraduate laboratories: a study of students' perceptions5

demonstrator are identical. Both circulate in the class and interact with students. However, the principal has the academic charge of the class and interacts with the class as a whole, for example, to introduce an experiment. The principal is also responsible for marking students' work associated with the laboratory class. Our recruitment and remuneration policies reflect the distinction between the principal and assistant demonstrators. The principals are drawn from non-career or pre-career physicists who either possess a PhD or are working towards it. The assistants are recruited from the ranks of postgraduate students, Honours students, and student peers. The peer demonstrators are themselves students majoring in the same type of degree as the students in the class. These peers recently completed, and excelled in, the subject (except for the physical science subject where only physics majors at Honours level or higher are recruited). To our knowledge, employing non-physics majors as peer demonstrators has not been previously reported in the context of a physics laboratory. In a general chemistry laboratory, McCreary et al. (2006) reported the use of advanced undergraduates as mentors to a small group of students in a laboratory-based workshop.

Towards the end of each semester, students were invited to take part in an anonymous online survey, in which they were questioned on a number of aspects of their subject. The survey was administered independently by the Planning and Quality Unit at UTS as part of a routine, institution-wide, subject evaluation program. Over 4 years (8 semesters, from 2011 to 2014), 1727 students participated in the survey, representing $34 \%$ of the total number of enrolled students. Of the respondents, $40 \%$ were engineering students, $43 \%$ were life sciences students, and $17 \%$ were physical sciences students. The number of respondents was generally consistent from semester to semester (the number of participants in the second semester being on average just $3 \%$ smaller than in the first semester). A notable exception was the first semester of 2013 when only one subject was surveyed. Due to course timetabling, the number of students in each subject changes markedly between semesters (the engineering cohort being most numerous in the first, and the life science cohort in the second semester).

The standard university subject survey was augmented with five questions relating specifically to the demonstrators (Table 1).

\begin{tabular}{ll}
\hline Shorthand & Survey item \\
\hline Help/P & The principal demonstrator was well prepared to help me with my work. \\
Help/A & The assistant demonstrator was well prepared to help me with my work. \\
Depth/P & The principal demonstrator encouraged me to think deeply about the experiments. \\
Depth/A & The assistant demonstrator encouraged me to think deeply about the experiments. \\
Feedback/P & The principal demonstrator gave me good feedback on my work. \\
\hline
\end{tabular}

Table 1. The demonstrator-focused survey items. In the shorthand form, "P" and "A" refer to the principal and assistant demonstrators, respectively. The respondents were asked to rate the statement on the Likert scale ranging from 5 for "strongly agree" to 1 for "strongly disagree". 
Non-physics peer demonstrators in undergraduate laboratories: a study of students' perceptions6

The survey data comprised the number of respondents, the sample mean of the Likert scores and the standard deviation for each of the laboratory classes surveyed. We used the pooled mean and the pooled standard error of the mean. Comparisons of means were carried out using the $t$-statistic expressed in terms of standard errors,

$$
t=\frac{\bar{x}_{1}-\bar{x}_{2}}{\sqrt{s_{e 1}^{2}+s_{e 2}^{2}}}
$$

where $\bar{x}$ is the mean, $s_{e}$ is the standard error, and the numerical subscript refers to the sets of classes being compared. The effective number of degrees of freedom was obtained by Welch-Satterthwaite equation (Welch 1947). The null hypothesis posited the equality of the means and was tested at 0.05 significance level $(p(<0.05))$. Where statistically significant differences between means are found, a measure of the effect size is also reported as the standardized mean difference or Cohen's $d$ value (Cohen 1977). All calculations were implemented in Excel (Microsoft Office 2010).

The focus of this study is the differentiation of student responses based on the separation in age, career choice, and background between the demonstrators and the students. As a proxy for the separation, we use the completed nominal number of years of post-secondary study in physics. As a reference, the separation among the students in the laboratory class is nil. The different categories of the separation are outlined in Table 2 .

\begin{tabular}{lr}
\hline Completed years of post-secondary study in physics & Category \\
\hline Nil (typical of students in the class) & $0 \mathrm{yrs}$ \\
Up to 1 completed year (typical of peer demonstrators) & $1 \mathrm{yrs}$ \\
3 completed years (typical of honours students) & $3 \mathrm{yrs}$ \\
4 to 7 completed years (typical of postgraduate students) & $4-7 \mathrm{yrs}$ \\
More than 7 completed years (typical of demonstrators possessing a PhD) & $>7 \mathrm{yrs}$ \\
\hline
\end{tabular}

Table 2. Categorization of demonstrator-student separation based on the number of completed years of post-secondary study of physics.

\section{Results and Discussion}

The response data set is multidimensional. It spans time, subject areas, as well as demonstrator characteristics. We first examine the chronological consistency of the data. Fig. 1 shows a plot of the mean Likert score averaged over the demonstrator items in the survey and over subjects. All the mean responses fall into the 3.6-3.9 interval, corresponding most closely to the discrete "agree" answer on the Likert scale. The standard errors are small reflecting the large sample sizes. Apart from 2011, small but statistically significant (at 0.05 significance level) differences in the means appear between the first and second semester of each year ( $2 \%$ difference in $2012,7 \%$ in 2013 and $6 \%$ in 2014). 
Non-physics peer demonstrators in undergraduate laboratories: a study of students' perceptions7

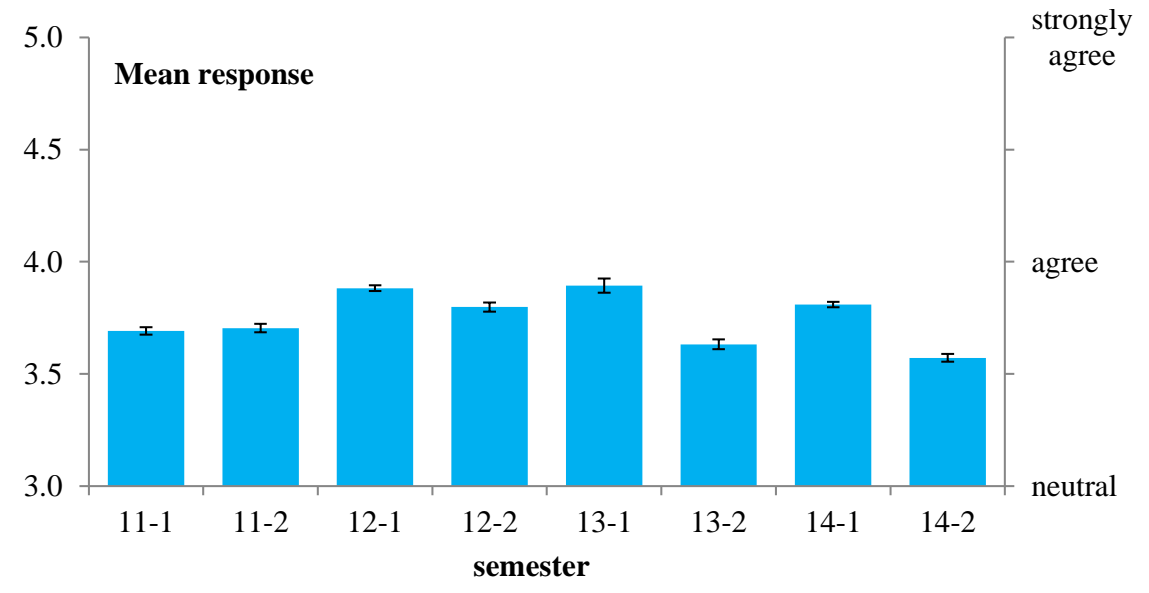

Figure 1. Mean response as a function of time, averaged over survey items and subjects. The error bars correspond to the standard error in the mean. The horizontal axis marks semesters as YY-S (for example, 11-1 stands for the first semester of 2011).

There are also significant differences in the mean, year to year, in the corresponding semester. For either semester, the response peaks in 2012 and gradually declines afterwards. It is not possible to ascribe this variation to, for example, changes in demonstrator training as both the student cohort and, to some extent, the demonstrator cohort varied markedly from year to year.

The semester variation within a year may be due to differences in the student cohort. In the first semester, on average, $61 \%$ are engineering students whereas in the second semester, $68 \%$ are life science students. Fig. 2 shows the mean response over each course cluster averaged over semesters and survey items. The mean responses from the engineering students and from the physical science students are statistically indistinguishable (at the 0.05 significance level) whereas the response from the life science students was $3 \%$ lower. The difference between the means is significant $(p<0.05)$ although the effect on the Likert scale is marginal (a tenth of a step between Neutral and Agree responses) and the effect size is small $(d=0.2)$. The distinction may be attributable to the greater similarity between engineering and physical science courses than between life science and either engineering or physical science course.

Fig. 3 shows the mean responses to specific survey statements, differentiated by the students' course category. Consider the response to the "Help" statement. The assistant demonstrators scored 0.2 (a fifth of the Likert unit) or $6 \%$ higher than the principal demonstrators in the engineering and life classes (the difference is statistically significant at $p<0.05$ with small effect size $d=0.2$ ). However, there is no significant difference between the two in physical sciences classes. It is possible that this reflects the presence of peer demonstrators in the former but not the latter. Somewhat paradoxically, the same results pertain to the responses to the "Depth" statement. Given the principal demonstrators' greater experience of physics, they would be expected to have the requisite knowledge to engage the students more deeply in the underlying science. The response to the "Feedback/P" statement is statistically indistinguishable from 
Non-physics peer demonstrators in undergraduate laboratories: a study of students' perceptions 8

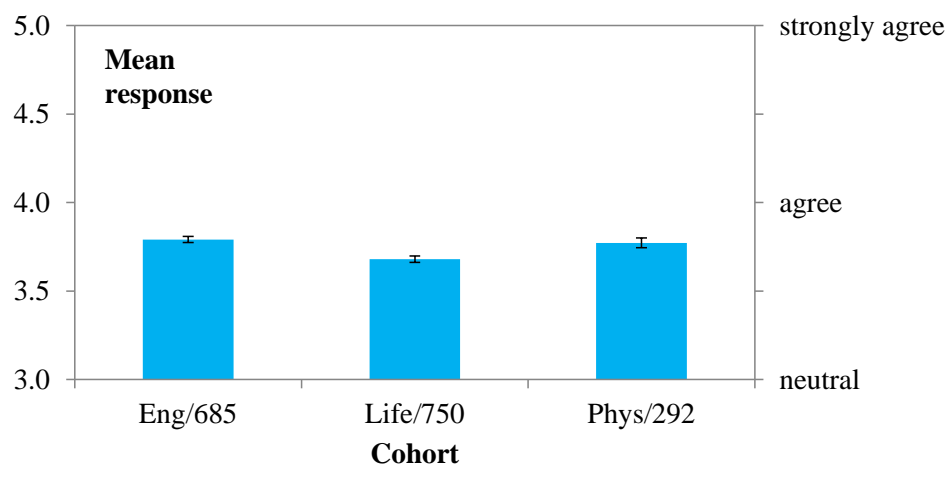

Figure 2. Mean response for three student cohorts (engineering, life sciences and physical sciences), averaged over survey items and semesters. The error bars correspond to the standard error in the mean. The horizontal axis marks the course taken by the students followed by the number of respondents.

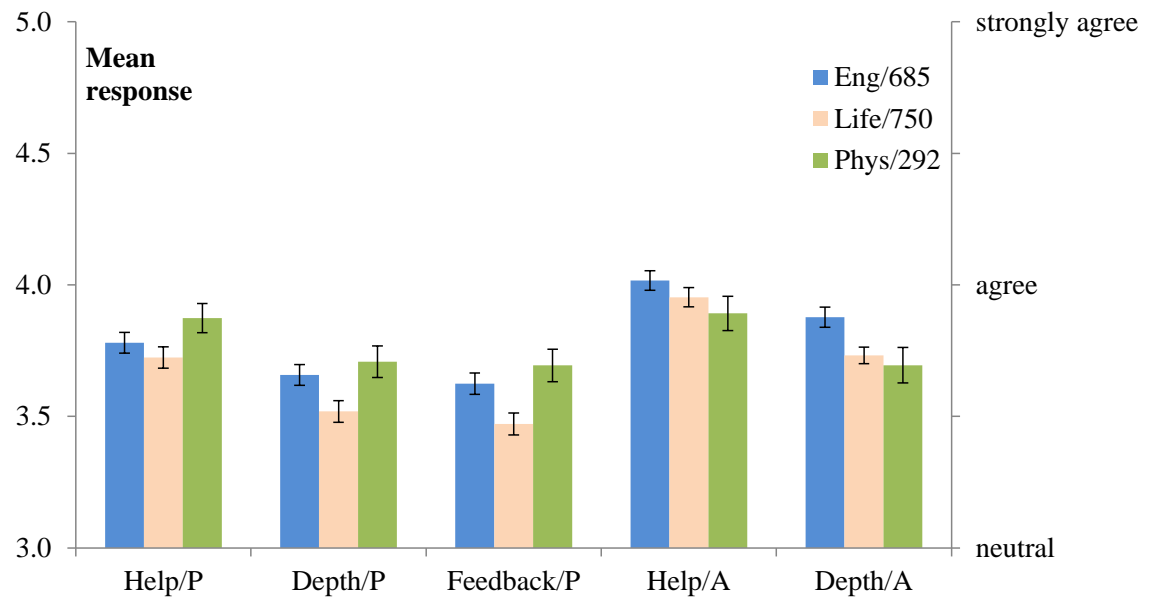

Figure 3. A comparison of mean responses in the three courses to each survey item, averaged over eight semesters. The error bars correspond to the standard error in the mean. The code to the categories on the horizontal axis is given in Table 1.

"Depth/P", suggesting that both categories reflect the perception of the quality of help received from the principal demonstrators. For both the principals and assistants, the "Help" scores are significantly higher than the "Depth" scores suggesting that students differentiate between the willingness to help and the quality of help received.

The mix of demonstrators at both the principal and assistant level prevents us from interpreting the differential response to principal and assistant demonstrators in terms of their background. We therefore recast the results in terms of the demonstrator-student separation categories defined above. Fig. 4 plots the mean Likert response as a function of the separation.

The plot shows a statistically significant decline in mean response with increasing separation for both statements, except that the data for the lowest two categories (corresponding to peer and Honours demonstrators) are not significantly different. The 
Non-physics peer demonstrators in undergraduate laboratories: a study of students' perceptions9

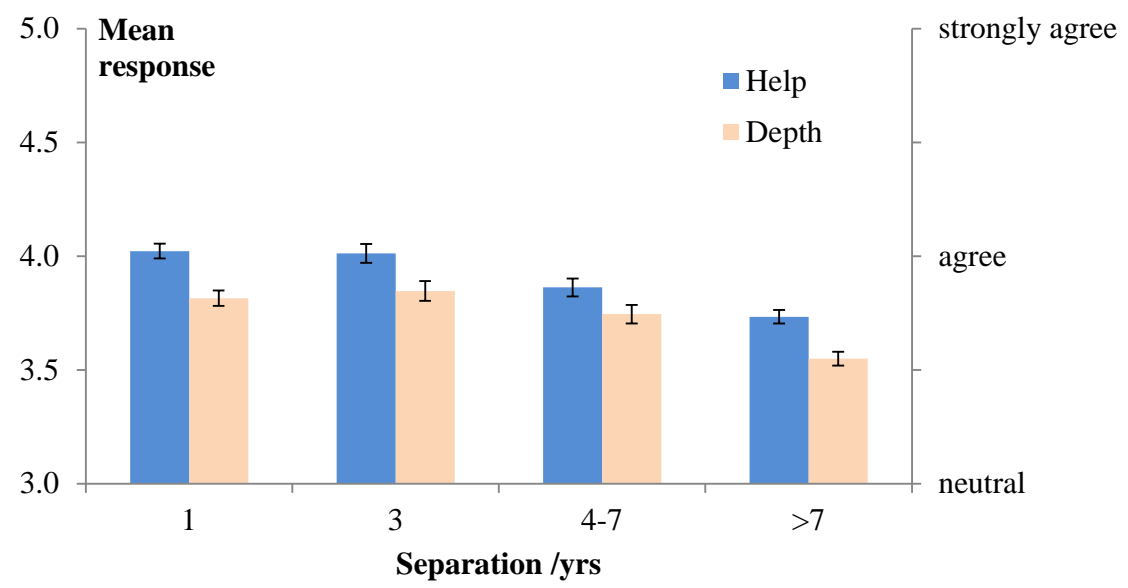

Figure 4. Mean response to the "Help" and the "Depth" statements, averaged over courses and semesters. The error bars correspond to the standard error in the mean. The horizontal axis marks the categories of separation between the demonstrators and the students as defined in Table 2 .

exception is interesting in that the peers and the Honours demonstrators are separated by 2 years of formal physics study whereas the difference between an Honours and a postgraduate demonstrator may only be 1 year. The response for the category consistent with the greatest post secondary experience of physics (demonstrator with $\mathrm{PhD}$ ) was 0.29 or $7.7 \%$ lower $(p<0.05, d=0.28)$ than the response for the category consistent with the least post secondary experience of physics (peer demonstrator). A similar result was found in comparing the $\mathrm{PhD}$ demonstrator and the Honours demonstrator $(0.28$, or $7.5 \%, d=0.27)$. The difference between the responses for the principal and the assistant demonstrators was less pronounced. With reference to the data of Fig. 3, the responses for the principal demonstrator was $5.0 \%$ lower than that for the assistant $(5.2 \%$ lower in the "Help" question and $4.9 \%$ lower in the "Depth" question). The discrepancy suggests that the separation is a more pertinent criterion than the demonstrator's role.

Gender preference may be relevant in interpreting the results. Gender bias in student evaluations has been documented (Basow 1995) and points particularly to a lower evaluation of female teachers by male students. Based on 2014 data, the majority of the engineering and physical science students were male (86\% and $73 \%$, respectively) whereas among the life science cohort, female students slightly outnumbered the male students (58\% to $42 \%$ ). Among the classes surveyed, $11 \%$ were guided by female principals and $25 \%$ by female assistants. However, there was a substantial difference in the female demonstrator participation when the figures were differentiated by subject into the life science and the non-life science cohorts. The proportions of female principals differed by only $2 \%$ but those of female assistants were substantially different. Females assistant demonstrators accounted for half the life-science classes but only $7 \%$ of the engineering/physical science classes. As many of the assistants were peer demonstrators, the proportion of females reflects the gender ratio in their majors.

Fig. 5 points to a lack of gender bias among the engineering/physical science 
Non-physics peer demonstrators in undergraduate laboratories: a study of students' perceptions10
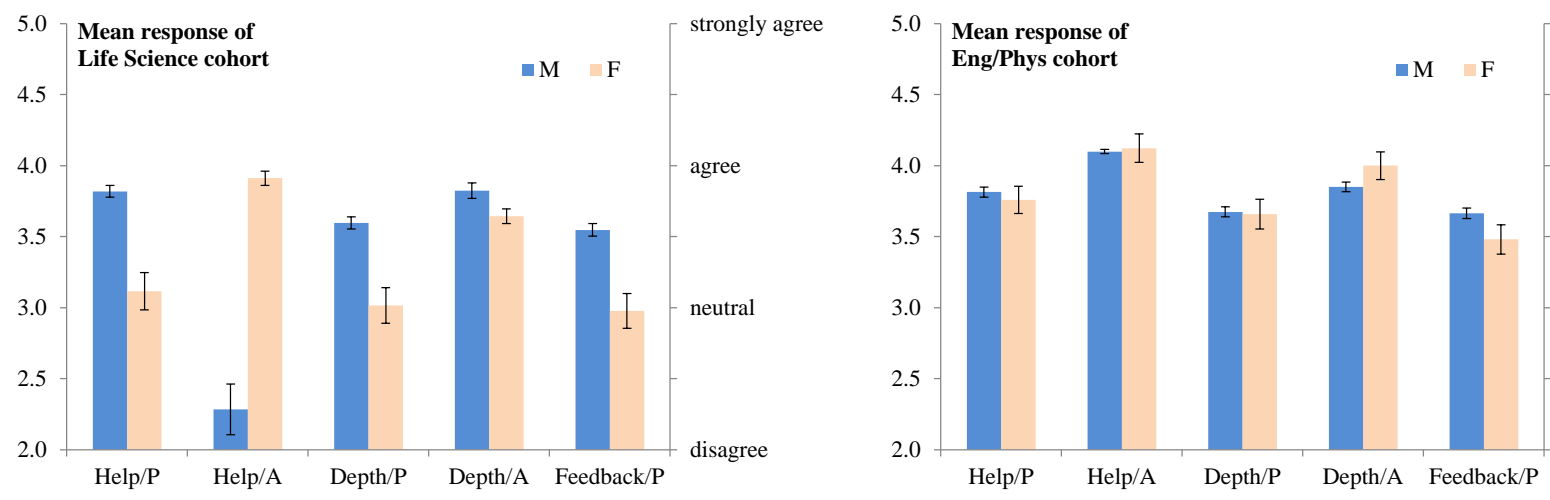

Figure 5. Variation of the mean response with the gender of the demonstrator for (left) life science, and engineering/physical science (right) students. The horizontal axis marks the five statements in the survey. The two series record response to male $(\mathrm{M})$ and female $(\mathrm{N})$ demonstrators. The error bars correspond to the standard error in the mean.

students (none of the male/female differences are statistically significant). However, among the life science students, for 4 out of 5 statements, the response appears to favour the male demonstrators. This is reversed in the case of the helpfulness statement for the assistant demonstrators, with the response to the female responses being $70 \%$ higher. This stems from an anomalously low score for male demonstrators. The results are indicative only. A more direct bias indicator would require the identification of the gender of individual students, which was not available.

While providing adequate statistical power to determine the presence of differences in response, the survey does not allow a detailed examination of student perceptions. A more nuanced interpretation requires the use of smaller scale tools, such as oneon-one interviews. We have expressed the difference in students' perceptions of the peer and non-peer demonstrators as a function of the separation between students and demonstrator measured in years of formal study of physics. What remains to be determined are the aspects of the interaction with students that are responsible for the observed differentiation of perception. Do the peer demonstrators interact less formally with the students? The peer demonstrator's familiarity with the students' courses enables them to identify where the physics will be used by the students. Does this knowledge allow peer demonstrators to bring a context and depth to their interaction with the students that the non-peer demonstrators cannot match? The survey items allow a degree of ambiguity. For example, in responding to the Help statement, were students focusing on the demonstrators's depth of knowledge or on the willingness to help? Although the study controlled for students choice of major, which is a proxy for their motivation to study physics, it did not control for individual differences in the motivation within the same major. These and other questions deserve to be the subject of a more intensive study. This study did not attempt to ascertain the demonstrators' perceptions of their interactions with students. An international study that examines the critical aspects of demonstrator-student interactions, employing small-scale tools 
and bringing in both the students' and demonstrators' perceptions, is currently under way. Identification and analysis of these aspects of interaction would inform the design of professional development programs for demonstrators.

\section{Conclusion}

A survey-based study of student perceptions of demonstrators in first-year physics laboratory classes was conducted spanning 4 years and more than 1700 students. The study was prompted by the introduction of non-physics major peer demonstrators. The results show that, compared to $\mathrm{PhD}$ demonstrators, the less experienced demonstrators (peer and Honours students) received 8\% higher scores in the perception of the demonstrator being well prepared to help and encouraging the student to think deeply about the experiment. Although the effect size is relatively low $(d=0.28)$, the absolute difference of between a third and a quarter of a Likert scale step is notable. The evidence clearly supports the proposition that the response to peer and Honours demonstrators was no less than that for the $\mathrm{PhD}$ demonstrators. The outcome points to the importance of the role of student-demonstrator proximity in age, career choice and background for the effectiveness of interactions between the demonstrators and students and validates the recruitment of peer demonstrators. An in-depth study is required to elucidate the reasons for the observed effects. It is intended that the study reported here, together with the in-depth study, will be used to inform the processes employed by tertiary education providers to recruit, develop and support laboratory demonstrators.

\section{Acknowledgements}

The authors wish to acknowledge the staff of the Planning and Quality Unit at UTS for the compilation of the survey data. The work described here was partly funded through the Office for Learning and Teaching, Sydney, Australia. The views expressed in this paper do not necessarily reflect the views of the Australian Government Office for Learning and Teaching.

\section{References}

Arendale D 2002 in D. B Lundell and L. L Higbee, eds, 'Histories of Developmental Education' University of Minnesota Minneapolis, MN pp. 15-27.

Basow S A 1995 J. Educ. Psychol. 87(4), 656.

Cohen J 1977 Statistical power analysis for the behavioral sciences Academic Press.

Cox A J and Junkin III W F 2002 Phys. Educ. 37(1), 37-44.

Crouch C H and Mazur E 2001 Am. J. Phys. 69(9), 970-977.

Dawson P, van der Meer J, Skalicky J and Cowley K 2014 Rev. Educ. Res. 84(4), 609639. 
Hensen K A and Shelley M C 2003 J. Coll. Student Dev. 44(2), 250-259.

Jaeger A J 2008 Academe 94(6), 42.

Jarrett L E, Takacs G and Ferry B 2010 Int. J. Innov. Sci. Math. Educ. 18(1).

Kendall K D and Schussler E E 2012 CBE-Life Sci. Educ. 11(2), 187-199.

Kirkup L, Pizzica J, Waite K and Srinivasan L 2010 Eur. J. Phys. 31(5), 1061.

McCreary C L, Golde M F and Koeske R 2006 J. Chem. Educ. 83(5), 804.

Psillos D and Niedderer H, eds 2002 Teaching and Learning in the Science Laboratory Kluwer.

Rice J W, Thomas S and OToole P 2009 'Tertiary science in the 21st century'.

URL: http://www.acds.edu.au/tlcentre/wp-content/uploads/2013/01/Rice09_Tertiaryscience-education-in-the-21st-century-final-report-2009.pdf (retrieved 7 May, 2015).

Roehrig G H, Luft J A, Kurdziel J P and Turner J A 2003 J. Chem. Educ. 80(10), 1206.

Roth W M and Roychoudhury A 1994 J. Res. Sci. Teach. 31(1), 5-30.

Seifert K, Fenster A, Dilts J A and Temple L 2009 CBE-Life Sci. Educ. 8(2), 147-153.

Von Aufschnaiter C and Von Aufschnaiter S 2007 Eur. J. Phys. 28(3), S51.

Welch B L 1947 Biometrika 34(1/2), 28-35.

Zepke N, Leach L and Butler P 2014 Higher Educ. Res. Dev. 33(2), 386-398. 